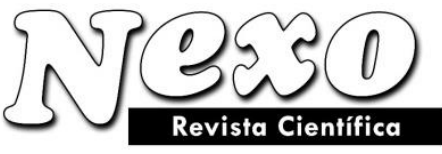

Vol. 29, No. 02, pp. 69-82/Diciembre 2016
ISSN-E 1995-9516

Universidad Nacional de Ingeniería

http://revistas.uni.edu.ni/index.php/Nexo

http://dx.doi.org/10.5377/nexo.v29i2.4576

\title{
EVALUACIÓN DE LA ESTABILIDAD DINÁMICA DEL FRESADO A TRAVÉS DEL ANÁLISIS DE PERFILES DE RUGOSIDAD
}

\section{DYNAMICAL STABILITY EVALUATION OF THE MILLING THROUGH ROUGHNESS PROFILES ANALYSIS}

\author{
Y. Sánchez Hechavarría ${ }^{1, *}$, M. Mariño Cala ${ }^{1}$ \\ ${ }^{1}$ Facultad de Ingeniería Mecánica Universidad de Oriente. Departamento de Manufactura y Materiales. \\ Patricio Lumumba s/n. Altos de Quintero. CP 90400. Santiago de Cuba. Cuba. \\ rysan@uo.edu.cu
}

(recibido/received: 01-Julio-2016; aceptado/accepted: 03-Septiembre-2016)

\begin{abstract}
RESUMEN
El objetivo de esta investigación es realizar la evaluación de la estabilidad dinámica del proceso de fresado de superficies inclinadas de acero endurecido AISI D6 con altas velocidades, a través del análisis de los perfiles de rugosidad en el sentido del avance. La evaluación de la estabilidad dinámica se basó en el análisis de los perfiles de rugosidad, la señal de la componente de la fuerza en el eje Y en el dominio del tiempo y la Raíz Media Cuadrática de las señales de fuerza en los tres ejes. La investigación demostró que es posible evaluar la estabilidad dinámica de un proceso de fresado a través del análisis de los perfiles de rugosidad, el obtenerse perfiles uniformes y menores valores de rugosidad superficial en los procesos más estables. Se demostró además que la rugosidad y la calidad superficial dependen en mayor medida de la estabilidad dinámica del proceso que de la velocidad de corte, lográndose en zonas de mayor estabilidad dinámica, aumentar la velocidad de avance y de corte con buenos resultados en la rugosidad superficial de la pieza fresada.
\end{abstract}

Palabras claves: Fresado, acero endurecido, fuerza de corte, rugosidad superficial, estabilidad dinámica.

\begin{abstract}
The objective of this research is to carry out the evaluation of the dynamic stability of inclined surface milling process of the hardened steel AISI D6 with high speeds, through the analysis of the roughness profiles in the sense of the advance. The evaluation of the dynamic stability was based in the analysis of the roughness profiles, the signal of the force component in the axis $\mathrm{Y}$ in time domain and the Root Mean Square of the signals of force in the three axes. The research demonstrated that it is possible to evaluate the dynamic stability of a milling process through the analysis of the roughness profiles, being obtained profiles uniforms and smaller values of superficial roughness in the most stable processes. It was also demonstrated that the roughness and the superficial quality depend in bigger measure of the dynamic stability of the process that of the cutting speed, being achieved in areas of more dynamic stability, to increase the advance speed and cut with good results in the superficial roughness of the milled piece.
\end{abstract}

Keywords: Milling, hardened steel, cutting force, roughness surfaces, dynamical stability. 


\section{Y. Sánchez-Hechavarría y M. Mariño Cala}

\section{INTRODUCCIÓN}

La detección del fenómeno de las vibraciones regenerativas es un aspecto muy importante a considerar en la obtención de una buena calidad superficial en la pieza, la reducción del ruido y la disminución de desgaste de la herramienta de corte (Lamraoui et al., 2015).

Ko y Shaw (2009) afirman que las vibraciones regenerativas que afectan el sistema permiten determinar el efecto regenerativo en la estabilidad dinámica del sistema y diferenciarlo del efecto de las vibraciones forzadas producidas por la frecuencia de entrada de dientes.

Dos caminos principales son seguidos para determinar y valorar el efecto de este fenómeno: la detección en línea y predicción. Las técnicas de detección en línea tratan de medir la inestabilidad mediante la señal captada por varios sensores, los más utilizados son: los sensores de fuerza, los acelerómetros y los micrófonos. Las técnicas de predicción simulan el sistema de mecanizado y tratar de anticipar el comportamiento vibratorio con el fin de calcular los parámetros óptimos (velocidad del husillo, profundidad de corte y así sucesivamente) para una operación determinada (Mandal, 2014).

Haythem (2011) plantea que las señales obtenidas mediante la detección en línea deben ser procesadas para extraer información como: la raíz media cuadrática, amplitud de la señal, frecuencia y tiempo. Los operadores de máquinas o un controlador numérico de máquina herramienta a continuación, pueden utilizar esta información para sugerir o ejecutar acciones correctivas apropiadas.

Las técnicas de predicción generalmente usan un modelo aproximado que consiste en analizar el comportamiento dinámico del sistema mediante un número de modos que se consideren representativos del sistema herramienta-cabezal-máquina, con las direcciones reales de desplazamiento de cada uno (Bediaga et al., 2004; Ganguli et al., 2006).

Pal Pandian et al., (2012) y Cao et al., (2014) utilizaron un modelo para calcular los desplazamientos considerando el sistema herramienta -portaherramientas - husillo como un sistema sencillo de dos grados de libertad en la dirección de X e Y, figura 1.9. De manera que la respuesta dinámica total del sistema es calculada mediante la suma de cada modo. La dinámica del fresado puede ser expresada mediante las ecuaciones diferenciales 1 y 2 .

$$
\begin{aligned}
& M_{x} \ddot{x}(t)+C_{x} \dot{x}(t)+K_{x} x(t)=F_{x}(t) \\
& M_{y} \ddot{y}(t)+C_{y} \dot{y}(t)+K_{y} y(t)=F_{y}(t)
\end{aligned}
$$

Donde, $\mathrm{M}, \mathrm{C}, \mathrm{K}$ son la masa, amortiguamiento y rigidez de la estructura para los modos respectivos, $\mathrm{F}_{\mathrm{x}}$ y $\mathrm{F}_{\mathrm{y}}$ son las proyecciones de la fuerza tangencial y radial en los ejes X e Y para cada modo.

Este modelo está basado en la dependencia de las vibraciones regenerativas de los coeficientes que ajustan la fuerza de cizallamiento, y que dependen del binomio herramienta - pieza, y de la dinámica de la máquina herramienta que varían con la variación de las velocidades de rotación del husillo. (Yue, 2006; Grossi et al., 2014).

Las posiciones son entonces determinadas mediante integración numérica en dependencia de la variación de la velocidad del husillo, y esta variación es aplicada para determinar los desplazamientos del sistema herramienta -portaherramientas - husillo. Los grados múltiples de libertad en cada dirección pueden ser acomodados sumando las contribuciones modales individuales (Grossi et al., 2014).

Nexo Revista Científica / Vol. 29, No. 02, pp. 69-82 / Diciembre 2016 


\section{Y. Sánchez-Hechavarría y M. Mariño Cala}

Una de las formas más empleadas para determinar las zonas de inestabilidad dinámica en el proceso de fresado es la construcción de las cartas de estabilidad mediante la determinación de los lóbulos de estabilidad que permiten obtener las profundidades de corte y velocidades de corte donde no se manifiesta el fenómeno de chatter. Para determinar los lóbulos de estabilidad se utiliza el modelo dinámico del proceso de fresado.

La simulación dinámica del proceso de fresado es más complicada que la de otros procesos de manufactura por arranque de viruta debido principalmente a que tanto la geometría como el espesor de viruta cambian constantemente, habitualmente hay varios bordes cortantes trabajando simultáneamente y a las no linealidades inherentes a este tipo de mecanizado. Por esto, el cálculo de la profundidad de corte límite de chatter regenerativo mediante el análisis en el dominio de frecuencias ha sido problemático en comparación con otros procesos continuos (Bediaga et al., 2004).

Los lóbulos de estabilidad son un gráfico donde se representa la profundidad de corte límite en función de la velocidad de rotación del husillo. Estos lóbulos establecen un límite entre profundidades de corte estables, para una velocidad de giro de la herramienta determinada. A partir de la construcción del gráfico de lóbulos, se pueden seleccionar las condiciones de corte apropiadas para evitar la aparición de vibraciones regenerativas (Gradišek et al., 2005).

La construcción de los diagramas de estabilidad se realiza mediante un barrido "inteligente" sobre la banda de las velocidades de rotación deseadas a diferentes profundidades de corte. Para cada binomio velocidad/profundidad de corte se calculan las fuerzas que actúan y se obtiene su espectro de frecuencias, al que se le realiza un filtrado selectivo de las frecuencias debidas al impacto de los dientes con objeto de aislar la frecuencia de las vibraciones regenerativas. Finalmente se impone un límite estático sobre el espectro para determinar la existencia de estas vibraciones en la señal correspondiente al binomio estudiado (Bediaga et al., 2004; Yue, 2006).

Obtener los diagramas de lóbulos de estabilidad presupone el conocimiento de la parte real de la función de respuesta de frecuencias (FRF) y de los coeficientes de amortiguamiento del sistema herramienta portaherramientas - husillo para determinar la profundidad de corte mínima y la frecuencia de las vibraciones regenerativas además se necesitan obtener valores precisos de la frecuencia natural, ya sea mediante la aplicación del método de elementos finitos o mediante ensayos de impacto, para determinar la relación entre la frecuencia natural $\left(f_{n}\right)$, la frecuencia de entrada de dientes (f) y la frecuencia de las vibraciones regenerativas.

Polli (2005) obtuvo perfiles de rugosidad uniformes, para procesos dinámicamente estables con f próxima a 3/4 de la frecuencia natural predomínate del sistema, Figura 1a, en los cuales los picos de rugosidad tienen una separación igual al avance por dientes y en algunos casos con la mitad de ese valor. Encontrándose también para estas condiciones los menores valores de rugosidad.

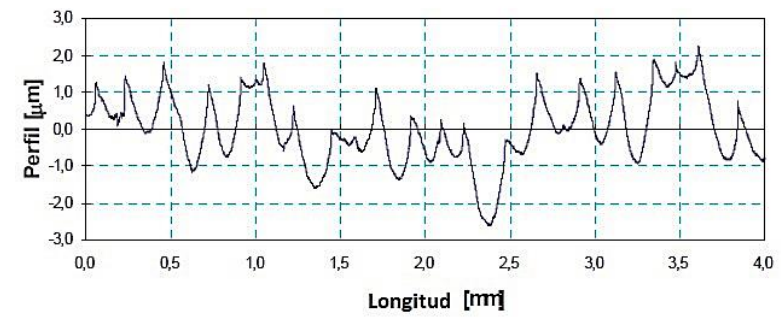

a)

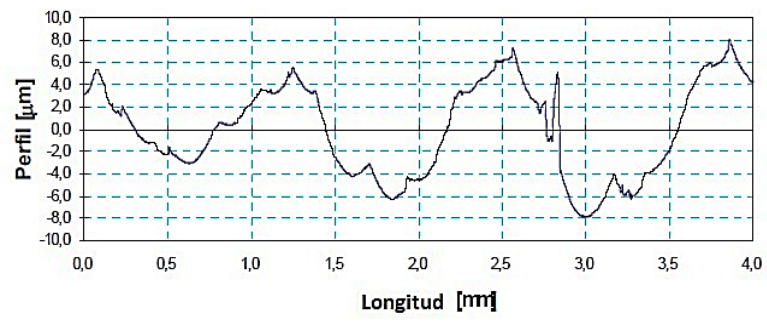

b)

Figura 1. Perfiles de rugosidad: a) Para un proceso de corte estable. b) Para un proceso de corte inestable. Fuente: adaptado de (Polli, 2005).

Nexo Revista Científica / Vol. 29, No. 02, pp. 69-82 / Diciembre 2016 
Para procesos dinámicamente inestables, donde la frecuencia de pasada de los dientes se corresponde con la mitad de la frecuencia natural predominante del sistema, se obtuvieron perfiles de rugosidad donde los picos tienen una separación de aproximadamente 6 veces el valor del avance por dientes con marcas visibles correspondientes al avance por dientes superpuestas a las marcas de mayor amplitud Figura $1 \mathrm{~b}$.

En el proceso de corte inestable, la amplitud de las señales de presión sonora obtenidas por Polli (2005) fueron mayores que para el proceso de corte estable $(n=16000 \mathrm{rev} / \mathrm{min})$, en el cual no se manifestaron picos de frecuencia cercanos a ningún modo de vibración asociado al sistema. Ya para los procesos de corte inestables $(\mathrm{n}=10500 \mathrm{rev} / \mathrm{min}$ y $\mathrm{n}=6750 \mathrm{rev} / \mathrm{min}$ ) se detectaron picos de frecuencia de gran amplitud muy cercanos a la frecuencia natural del sistema.

Quintana (2009) y Mendes de Aguiar et al., (2015) observaron una relación entre la rugosidad teórica de la viruta y la deflexión de la herramienta en el eje Y, concluyendo que el aumento de la fuerza de corte y de los desplazamientos producen peores perfiles de rugosidad y texturas superficiales en las piezas fresadas.

Gökkaya (2010) encontró una relación directa entre la componente radial de la fuerza de corte y el valor de la rugosidad media aritmética Ra durante el fresado frontal de una pieza de aluminio aleado AA2014 (T4) con altas velocidades de corte. Determinando además la influencia de la velocidad de corte en la fuerza de corte y la rugosidad superficial. En el estudio realizado, empleó velocidades de corte de 200, 300, 400 y 500 $\mathrm{m} / \mathrm{min}$. Los resultados de este estudio muestran que la rugosidad superficial aumenta con el aumento de la velocidad de corte de hasta $300 \mathrm{~m} / \mathrm{min}$ y luego va disminuyendo como se observa en la Figura 2.

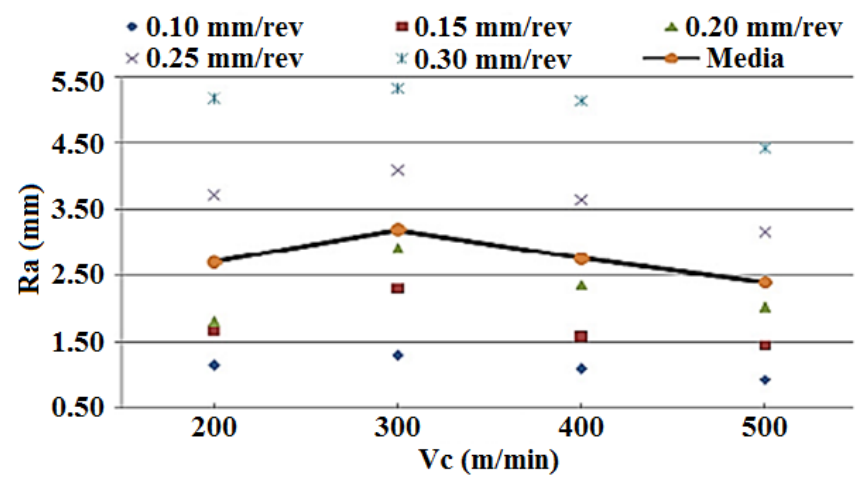

Figura 2. Valor promedio de la rugosidad superficial en función de la velocidad de corte. Fuente: adaptado de (Gökkaya, 2010).

Gok et al., (2012) determinaron durante el proceso de fresado de una superficie curva de acero EN X40CrMoV5-1 con dureza aproximada de 54 HRC, que los peores valores de acabado superficial estaban relacionados con los mayores niveles de presión sonora, lo cual está asociado según estos autores, a un aumento de los valores de las fuerzas de corte y a la presencia de vibraciones regenerativas debido a la mayor variación entre los cortes subsecuentes de la herramienta, que están relacionados con el aumento de los desplazamientos en las direcciones $\mathrm{X}$ e $\mathrm{Y}$.

Wojciechowski (2011) realizó un estudio para determinar la correspondencia que existe entre los desplazamientos de la herramienta en Y y las señales de fuerza normal a esta superficie. En el mismo se determinó, mediante un sensor láser, la deflexión de la herramienta en el eje Y durante el fresado de una pieza de superficie inclinada a $90^{\circ}$ con respecto a la mesa de la máquina y las fuerzas de corte mediante un dinamómetro piezoeléctrico. El material de la pieza fresado fue el acero $55 \mathrm{NiCrMoV} 6$ con $55 \mathrm{HRC}$ de dureza. 
Este autor determinó que existe una relación directa entre los picos de los desplazamientos en Y (deflexión) y los picos de la fuerza de corte, como se observa en la Figura 3, ambos con una longitud de onda de 0,6 $\mathrm{mm}$, que para este estudio se correspondió con el valor del avance para cada revolución del husillo.

Al medir la rugosidad superficial de la pieza este autor observó que las pequeñas irregularidades correspondientes al valor de la rugosidad superficial tenían el mismo comportamiento en amplitud y longitud que la señal de fuerza normal a la superficie fresada y la deflexión de la herramienta en Y, existiendo una correspondencia cualitativa entre el curso temporal de la fuerza normal a la superficie y el perfil de rugosidad de la misma demostrándose una clara relación entre la variación de la fuerza máxima instantánea y el perfil de rugosidad generado. Determinó además, sobre la base de los resultados obtenidos en este estudio, el factor que más influencia tiene sobre el perfil de rugosidad de la superficie es la deflexión radial de la herramienta. Directamente relacionada con el valor de la componente radial de la fuerza de corte y con el periodo de revolución de la herramienta.

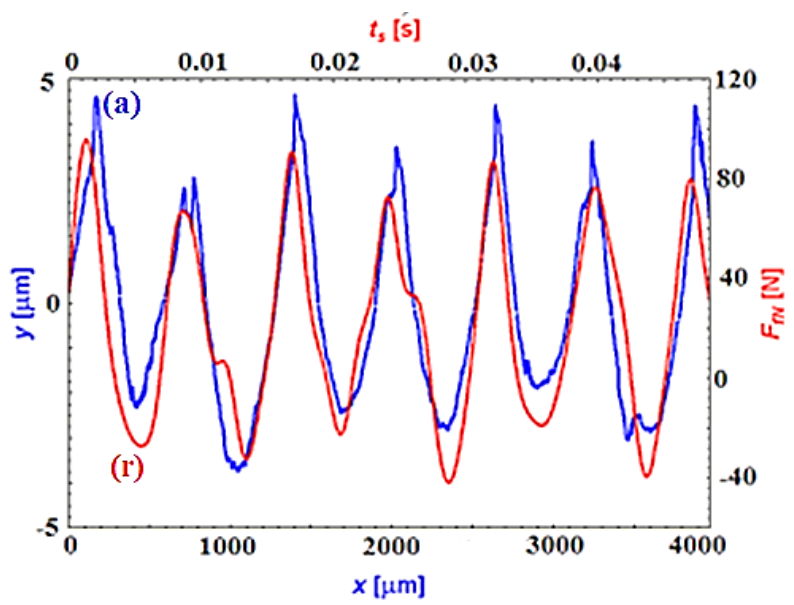

Figura 3. Perfil de rugosidad (azul) superpuesto al perfil de la fuerza normal a la superficie (rojo). Fuente: adaptado de (Wojciechowski, 2011).

Wojciechowski et al., (2013) determinaron que durante el fresado de una superficie inclinada de acero $\mathrm{X} 153 \mathrm{CrMoV} 12$ con una dureza de $60 \mathrm{HRC}$, las variaciones de las vibraciones y de las fuerzas de corte debido a la variación del ángulo de inclinación tienen una marcada influencia en la rugosidad superficial $\mathrm{Rz}$, aumentando su valor al aumentar $\theta$ y manteniendo constantes $\mathrm{V}_{\mathrm{C}}, \mathrm{a}_{\mathrm{p}} \mathrm{y} \mathrm{s}_{\mathrm{z}}$. Un aumento de $\theta$ produce una disminución de la fuerza axial y un aumento de la fuerza radial ocasionando una mayor deflexión de la herramienta de corte y por ende un aumento de las vibraciones, variables que están directamente asociadas a la estabilidad dinámica del proceso.

El objetivo de esta investigación es realizar el análisis de la estabilidad dinámica del proceso de fresado de superficies inclinadas a través del análisis de los perfiles de rugosidad en el sentido del avance, de la fuerza de corte en el eje Y y de las RMS de las señales de fuerza en los tres ejes.

\section{MATERIALES Y MÉTODOS}

Los experimentos fueron realizados en un centro de mecanizado vertical MORI SEIKI SV 40 con rotación máxima del husillo de $12000 \mathrm{rpm}$ y potencia principal de $22 \mathrm{~kW}$. Los cuerpos de prueba fueron fabricados con acero AISI D6 endurecido, el cual fue escogido debido a sus aplicaciones en la fabricación de matrices de corte y embutido en frío. La dureza del material utilizado para fabricar el cuerpo de prueba es de $62 \mathrm{HRC}$.

Nexo Revista Científica / Vol. 29, No. 02, pp. 69-82 / Diciembre 2016 
El acero AISI D6 es un acero aleado con alto contenido de carbono y de cromo, con tungsteno en su composición química. Este material tiene una alta tensión de compresión, alta resistencia al desgaste, alta dureza superficial y buena dureza. Las propiedades mecánicas y la composición química s de este material se muestran en las tablas 1 y 2 . En la tabla $1, \delta$ es la elongación del material, $\sigma_{C}$ es la tensión de compresión y E es el módulo de elasticidad.

Tabla 1. Propiedades mecánicas del acero AISI D6

\begin{tabular}{|c|c|c|c|}
\hline Dureza HRC & $\delta(\%)$ & $\sigma_{\mathrm{C}}(\mathrm{MPa})$ & $\mathrm{E}(\mathrm{GPa})$ \\
\hline Máx. 62 & $30-32$ & 1320 & 194 \\
\hline
\end{tabular}

Tabla 2. Composición química del acero herramental AISI D6.

\begin{tabular}{|c|c|c|c|c|c|c|c|c|c|}
\hline Elementos & $\mathrm{C}$ & $\mathrm{Mn}$ & $\mathrm{P}$ máx. & $\mathrm{S}$ máx. & $\mathrm{Si}$ & $\mathrm{Cr}$ & $\mathrm{W}$ & $\mathrm{V}$ & $\mathrm{Fe}$ \\
\hline$\%$ en masa & 2,1 & 0,4 & 0,03 & 0,03 & 0,3 & 11,5 & 0,7 & 0,2 & balance \\
\hline
\end{tabular}

El fresado horizontal ascendente es más favorable que el vertical descendente ya que determina una disminución del parámetro Ra en el sentido del avance y del incremento lateral y niveles más bajos de vibraciones (Toh, 2004; Pivetta, 2005; Cosma, 2011). Sin embargo esta estrategia de corte también es poco usada en la elaboración de moldes con cavidades estrechas o complejas debido a que la herramienta debe comenzar el proceso de corte desde el fondo de la cavidad hacia arriba. Por lo que se seleccionó para el proceso de fresado una estrategia horizontal descendente (Figura 4a) con la cual se obtienen buenos valores de rugosidad en el sentido del avance en detrimento de la rugosidad en el sentido de la alimentación (perpendicular al avance). El ángulo de inclinación de la superficie fresada ( $\theta$ ) durante los ensayos fue de $45^{\circ}$.

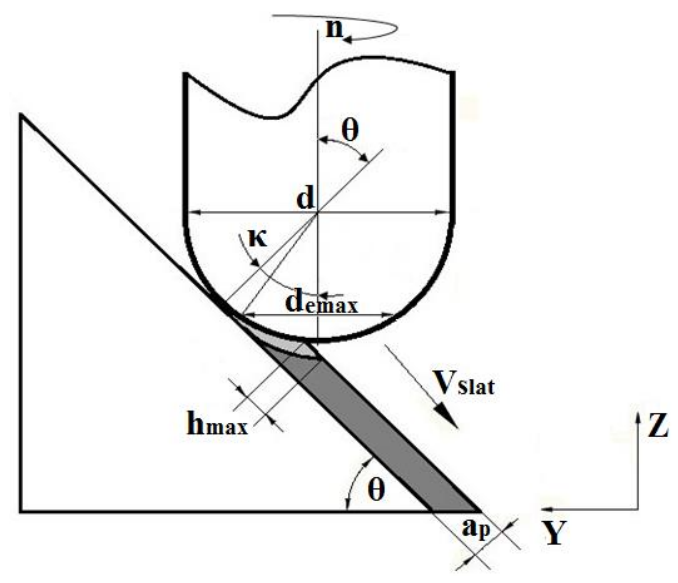

a)

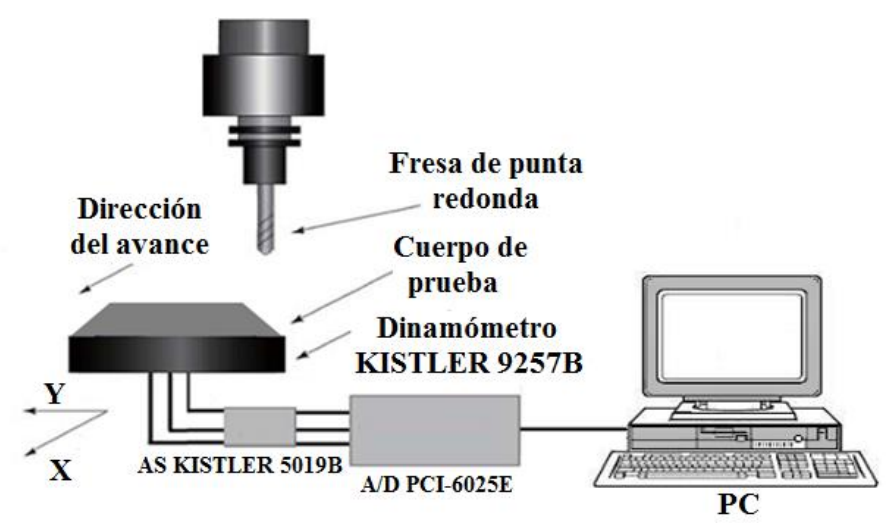

b)

Figura 4. a) Estrategia de corte horizontal descendente. b) Instalación experimental para el monitoreo de las señales de fuerza de corte.

Para los ensayos se usaron fresas de espiga de punta redonda SANDVIK COROMANT R216F-16 40 E-L de $16 \mathrm{~mm}$ de diámetro. Los calzos intercambiables son de la clase P10A (ISO H10) con recubrimiento TiAlN. Para el montaje de las pastillas se usaron dos portaherramientas de iguales dimensiones: uno de acero R216F-16A16S-063 (PH1) y otro de metal duro con extremo de acero R216F-16A16C-063 (PH2). Los mismos fueron fijados en voladizo con una longitud de $70 \mathrm{~mm}$. El proceso de corte se realizó en seco.

Nexo Revista Científica / Vol. 29, No. 02, pp. 69-82 / Diciembre 2016 
Para la selección del criterio de fin de vida de las herramientas se debe que tener en cuenta que las operaciones se realizaran en un régimen de acabado donde el requisito fundamental es conseguir buen acabado superficial y dimensional además de tolerancias pequeñas. Para estas operaciones el desgaste de flanco VB no debe exceder de 0,2 $\mathrm{mm}$ para calidades de IT7 y $0,3 \mathrm{~mm}$ para calidades de IT8 (Diniz et al., 2010). El desgaste de flanco máximo $\mathrm{VBB}_{\text {Máx }}$ se fijó en 0,2 $\mathrm{mm}$. En la tabla 3 se muestran las condiciones experimentales usadas en los ensayos.

Tabla 3: Condiciones de corte para los ensayos.

\begin{tabular}{|c|c|c|}
\hline $\mathrm{Vc}(\mathrm{m} / \mathrm{min})$ & $\mathrm{n}_{\text {efect }}(\mathrm{rpm})$ & $\mathrm{f}(\mathrm{Hz})$ \\
\hline 250 & 5354 & 178,5 \\
\hline 266,3 & 5703 & 190,1 \\
\hline 360 & 7710 & 257 \\
\hline 383,2 & 8213 & 273,8 \\
\hline 420 & 8995 & 299,8 \\
\hline 447,5 & 9583 & 319,4 \\
\hline
\end{tabular}

El avance por dientes $\left(f_{z}\right)$ de la herramienta es de $0,15 \mathrm{~mm} /$ diente y la profundidad de corte radial utilizada en los ensayos $\left(\mathrm{a}_{\mathrm{e}}\right)$ es igual $0,15 \mathrm{~mm}$.

Los perfiles de rugosidad se obtuvieron utilizando un rugosímetro portátil Mitutoyo, modelo SJ-201P, montado en un trazador de altura, lo que posibilita la inclinación del rugosímetro con relación a la superficie fresada. La adquisición del perfil de rugosidad se logró mediante el programa SJ-201Excel. La rugosidad fue evaluada utilizando los parámetros de rugosidad $\mathrm{R}_{\mathrm{a}} \mathrm{y} \mathrm{R}_{\mathrm{q}}$ en dos direcciones: transversal y longitudinal a la dirección del avance.

Para obtener la Función de Respuesta de Frecuencia $(F R F)$ de los portaherramientas, fueron realizados análisis modales para las dos combinaciones experimentales (con el portaherramientas de acero PH1 y con el de metal duro PH2). Para esto, fue fijado en el extremo del portaherramientas un acelerómetro uniaxial PCB PIEZOTRONICS 352C33, conectado a un acondicionador de señales PCB482B05 con ganancia unitaria y sin filtro. Por medio de un martillo de acero con punta de plástico, los portaherramientas fueron excitados mediante un impulso no controlado aplicado paralela y perpendicularmente a los ejes X e Y.

La captación y procesamiento de las señales de $F R F s$ se realizó mediante un acondicionador de señales multicanal SCIENTIFIC ATLANTA SD 385 conectado al acelerómetro uniaxial y que utiliza un procesador de señales basado en la Transformada Rápida de Fourier ( FFT). De esta forma se obtuvieron las funciones de transferencia $H(\omega)$ para los dos portaherramientas.

Para la medición de las fuerzas en las tres direcciones (X, Y, Z), fue fijado un dinamómetro Kistler 9257B en la mesa da máquina, colocándose el cuerpo de prueba sobre la base superior del mismo. Con este tipo de basificación se puede considerar la pieza como un cuerpo rígido. El dinamómetro está conectado a un acondicionador de señales Kistler 5019B y la adquisición de los datos se realizó mediante de una placa de adquisición de señales A/D National Instruments PCI-6025E, con salida hacia un computador que empleó el software LabView 8.5 para procesar los resultados. En la Figura $4 \mathrm{~b}$ se muestra un esquema de la instalación experimental utilizada en el monitoreo on-line de las señales de fuerza de corte.

En el dominio del tiempo fue evaluada la Raíz Media Cuadrática $(R M S)$ de la señal, tomado como parámetro estático a partir de la ecuación 3.

Nexo Revista Científica / Vol. 29, No. 02, pp. 69-82 / Diciembre 2016 


$$
R M S=\sqrt{\frac{1}{\mathrm{~N}_{\mathrm{p}}} \cdot \sum_{\mathrm{i}=1}^{\mathrm{N}} \mathrm{x}_{\mathrm{i}}^{2}}
$$

Donde $\mathrm{N}_{\mathrm{p}}$ es la cantidad de puntos adquiridos y $\mathrm{x}_{\mathrm{i}}$ e la variable de la señal.

El análisis de la $R M S$ de las componentes en los ejes $\mathrm{F}_{\mathrm{Y}}, \mathrm{F}_{\mathrm{Y}}$ y $\mathrm{F}_{\mathrm{Z}}$ de la fuerza de corte nos permite determinar la amplitud y magnitud de las señales fuerza en el dominio del tiempo.

\section{RESULTADOS Y DISCUSIÓN}

En la Figura 5a se muestra la rugosidad superficial media en función de la velocidad de rotación del husillo para un corte concordante durante el mecanizado de una superficie inclinada a $45^{\circ}$ para el portaherramientas PH1.

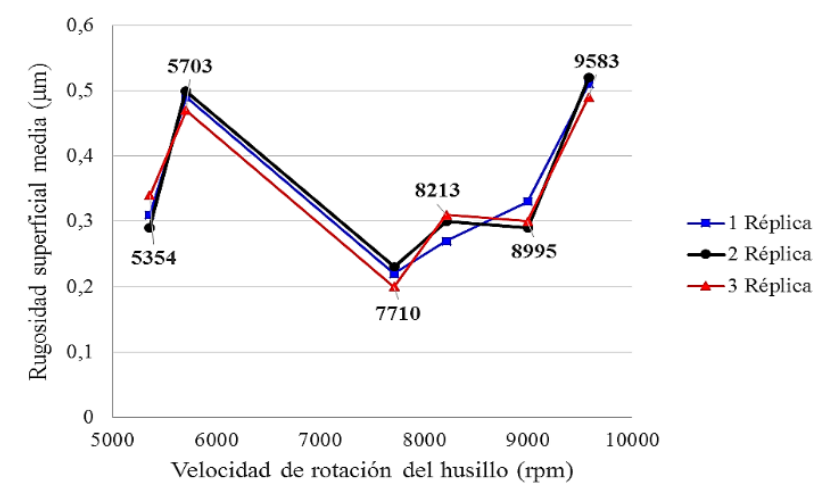

a)

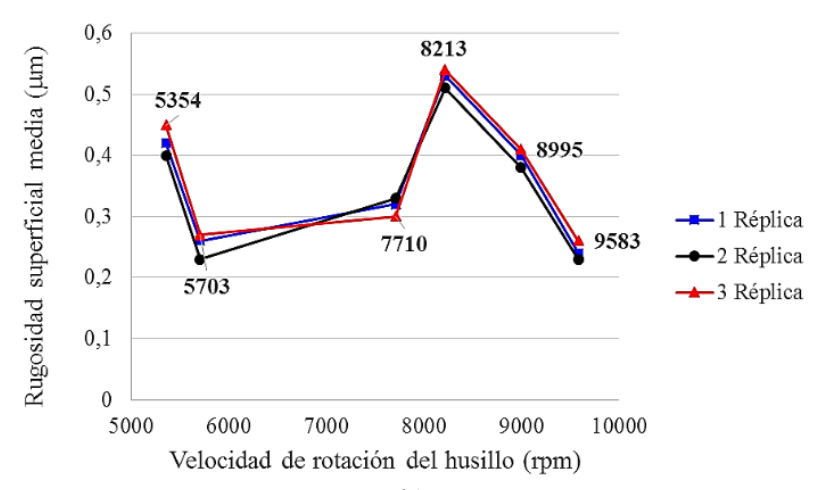

b)

Figura 5. Rugosidad superficial media en función de la velocidad de rotación del husillo. a) Portaherramientas $\mathrm{PH} 1 . \mathrm{VBB} \approx 0,180 \mathrm{~mm}$. b) Portaherramientas $\mathrm{PH} 2 . \mathrm{VBB} \approx 0,180 \mathrm{~mm}$.

Analizando la Figura 5a se evidencia que la variación de la rugosidad superficial no está en función de la variación de la velocidad de avance, los mayores valores de rugosidad se identifican en velocidades de corte donde los armónicos de la f se aproximan al modo de vibración 1 (frecuencia: $956 \mathrm{~Hz}$ ). Para la velocidad de corte de 266,3 $\mathrm{m} / \mathrm{min}$ cuyo armónico $5(f 5=952 \mathrm{~Hz})$ y para la velocidad de corte de $447,5 \mathrm{~m} / \mathrm{min}$ con armónico 3 ( $f 3=958,3 \mathrm{~Hz}$ ), los más próximos a la frecuencia modal 1 , se obtienen los peores resultados de rugosidad superficial media $\mathrm{Ra}$; los mejores resultados de rugosidad superficial media se obtienen para velocidades de corte de $360 \mathrm{~m} / \mathrm{min}(f 3=771 \mathrm{~Hz})$ y $383,2 \mathrm{~m} / \mathrm{min}(f 3=821,4 \mathrm{~Hz})$.

Tabla 4. Frecuencia de entrada de dientes y armónicos cercanos a la banda de frecuencias de la frecuencia modal 1 .

\begin{tabular}{|c|c|c|c|}
\hline $\mathrm{V}_{\mathrm{C}}(\mathrm{m} / \mathrm{min})$ & $\mathrm{n}_{\text {efect }}(\mathrm{rpm})$ & $\mathrm{f}(\mathrm{Hz})$ & Armónicos $(\mathrm{Hz})$ \\
\hline 250 & 5354 & 178,5 & $892,5 \mathrm{f5}$ \\
\hline 266,3 & 5703 & 190,1 & $952,5 \mathrm{f5}$ \\
\hline 360 & 7710 & 257 & $771,0 \mathrm{f3}$ \\
\hline 383,2 & 8213 & 273,8 & $821,4 \mathrm{f3}$ \\
\hline 420 & 8995 & 299,8 & $899,4 \mathrm{f3}$ \\
\hline 447,5 & 9583 & 319,4 & $958,3 \mathrm{f3}$ \\
\hline
\end{tabular}

En la tabla 4 se muestran las frecuencias de entrada de dientes y los armónicos más cercanos a la frecuencia modal 1 para los dos portaherramientas PH1 $(956 \mathrm{~Hz})$ y PH2 $(841 \mathrm{~Hz})$.

Nexo Revista Científica / Vol. 29, No. 02, pp. 69-82 / Diciembre 2016 
Según los datos mostrados en la Figura 5b se comprueba que cuando se mecaniza con el portaherramientas de metal duro PH2 los mayores valores de rugosidad superficial media se verifican en velocidades de corte donde los armónicos de la f se aproximan al modo de vibración 1 (frecuencia: $841 \mathrm{~Hz}$ ). El valor más bajo de rugosidad superficial media Ra durante el mecanizado con el portaherramientas $\mathrm{PH} 2$ se verifica para la velocidad de corte de $383,2 \mathrm{~m} / \mathrm{min}\left(\mathrm{n}_{\text {efect }}=8213 \mathrm{rpm}, \mathrm{f}=273 \mathrm{~Hz}\right.$ y $\left.f 3=821 \mathrm{~Hz}\right)$ que tiene su armónico 3 muy próximo a la frecuencia modal del modo 1 . Los mejores resultados se obtienen para velocidades de corte de 266,3 $\mathrm{m} / \mathrm{min}(f 5=952,5 \mathrm{~Hz})$ y 447,5 m/min $(f 3=958,3 \mathrm{~Hz})$, cuyos armónicos están más alejados de la frecuencia modal 1 del portaherramientas $\mathrm{PH} 2$.

En la Figura 6a se muestra el perfil de rugosidad en el sentido del avance obtenido durante el fresado de la superficie con $\mathrm{V}_{\mathrm{C}}=447,5 \mathrm{~m} / \mathrm{min}(\mathrm{n}=9583 \mathrm{rpm} ; \mathrm{f} 3=958,3 \mathrm{~Hz})$ empleando el portaherramientas de metal duro PH2. Se evidencia que para estas condiciones se obtiene un proceso de corte estable con marcas regulares en la superficie de la pieza que son propias de un proceso dinámicamente estable. En el perfil de rugosidad en el sentido del avance se observa que las marcas dejadas por la herramienta coinciden con el valor del avance $\left(\mathrm{s}=2 \mathrm{~S}_{\mathrm{Z}}\right)$ lo que muestra una estabilidad en el proceso de corte ya que la herramienta deja una marca definida por cada revolución del husillo. Estos perfiles son uniformes en el espaciamiento de los picos de rugosidad y en la altura de los mismos.

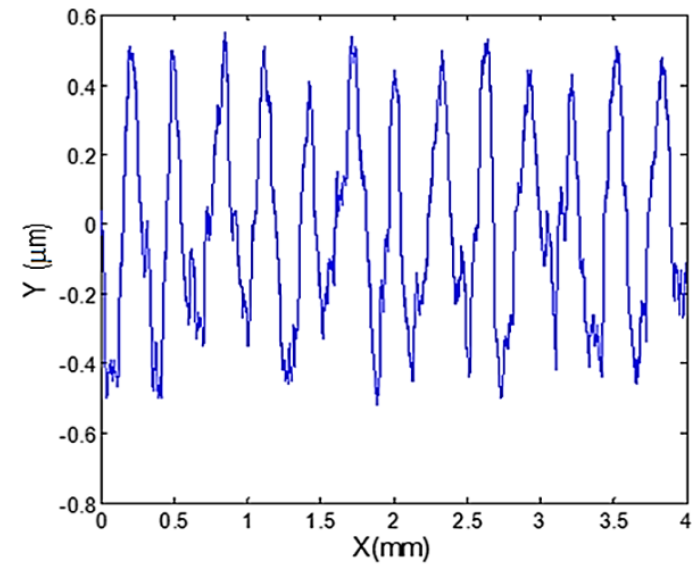

a)

$$
\mathrm{Ra}=0,24 \mu \mathrm{m} \quad \mathrm{Rq}=0,28 \mu \mathrm{m}
$$

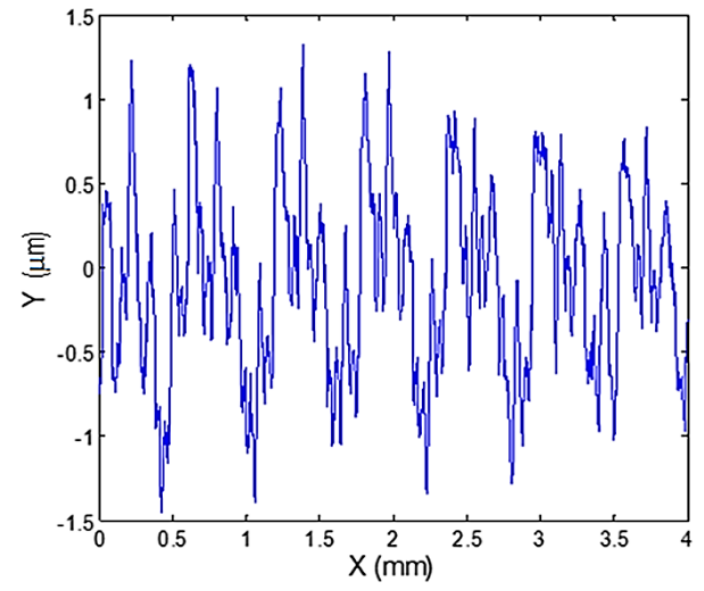

b)

$$
\mathrm{Ra}=0,51 \mu \mathrm{m}
$$$$
\mathrm{Rq}=0,61 \mu \mathrm{m}
$$

Figura 6. Perfil de rugosidad en el sentido del avance. a) Portaherramientas $\mathrm{PH} 2 . \mathrm{V}_{\mathrm{C}}=447,5 \mathrm{~m} / \mathrm{mim}$. b) Portaherramientas PH1. $\mathrm{V}_{\mathrm{C}}=447,5 \mathrm{~m} / \mathrm{mim}$.

En la Figura $6 \mathrm{~b}$ se muestra el perfil de rugosidad obtenido durante el fresado con $\mathrm{V}_{\mathrm{C}}=447,5 \mathrm{~m} / \mathrm{min}$ y portaherramientas PH1 ( $\mathrm{n}=9583 \mathrm{rpm}$; $\mathrm{f}=958,3 \mathrm{~Hz}$ ). En el mismo se observa que las marcas dejadas por la herramienta de corte, correspondientes al doble del valor del avance, siendo irregulares en toda la longitud de medición de la rugosidad. Estas irregularidades en el perfil de rugosidad indican inestabilidad dinámica en el proceso de fresado. En el perfil de rugosidad mostrado en la Figura 6a tienen una separación de aproximadamente 2 veces el valor del avance por dientes con marcas visibles correspondientes al valor del avance por dientes superpuestas a las marcas de mayor amplitud.

$\mathrm{Si}$ analizamos las señales de la componente en el eje $\mathrm{Y}$ de la fuerza en el dominio del tiempo $\left(\mathrm{F}_{\mathrm{Y}}\right)$ obtenidas durante el fresado con velocidad de corte de 447,5 m/min mostrados en la Figura 7a, podemos observar que los valores máximos instantáneos de la fuerza de corte para cada diente son uniformes con un periodo igual al tiempo que tarda la fresa en dar una revolución. Las variaciones del valor máximo de $F_{Y}$ e los dientes consecutivos, observados en las señales obtenidas con ambos portaherramientas, se debe al fenómeno de deflexión radial de la herramienta.

Nexo Revista Científica / Vol. 29, No. 02, pp. 69-82 / Diciembre 2016 


\section{Y. Sánchez-Hechavarría y M. Mariño Cala}

Las irregularidades mostradas en el perfil de rugosidad obtenido durante el fresado con el portaherramientas PH1 y velocidad de corte de 447,5 m/min (Figura $7 b$ ) tienen su origen en el aumento del valor máximo de $\mathrm{F}_{\mathrm{Y}}$ como se observa en la Figura 8b, lo que origina un mayor desplazamiento en Y y una mayor deflexión radial.

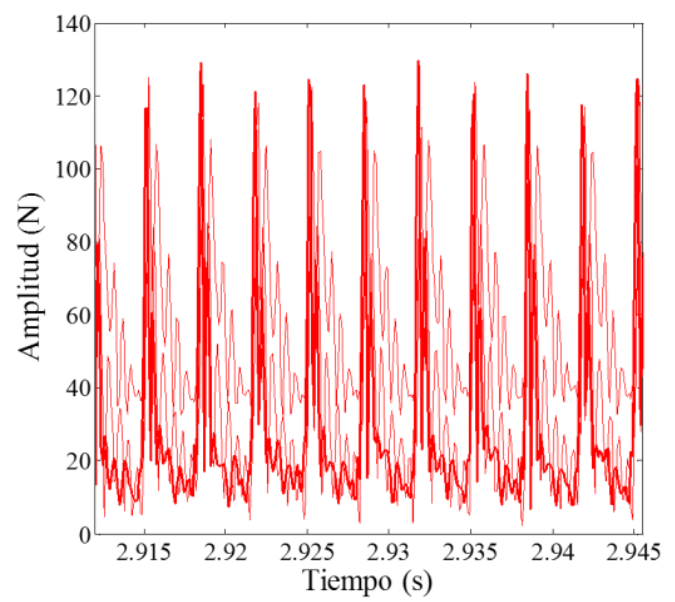

a)

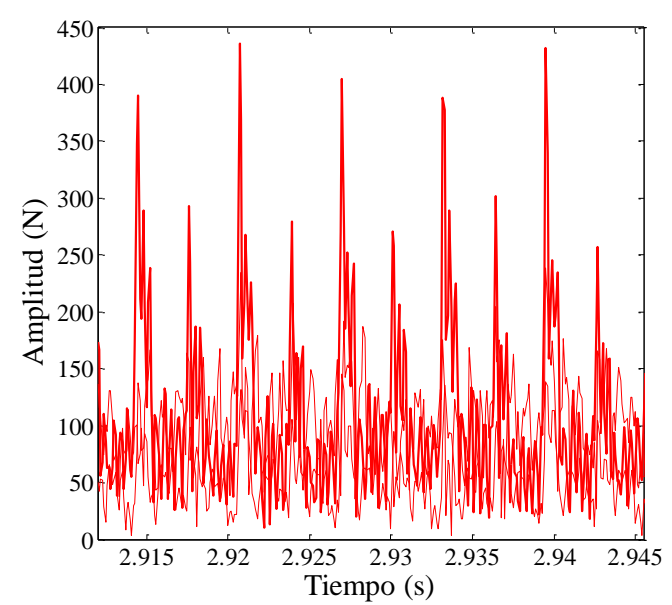

b)

Figura 7. Señales de fuerza en el dominio del tiempo. a) Portaherramientas $\mathrm{PH} 2 . \mathrm{V}_{\mathrm{C}}=447,5 \mathrm{~m} / \mathrm{min}$ y $\mathrm{VB} \approx 0,18$ mm. b) Portaherramientas PH1. $\mathrm{V}_{\mathrm{C}}=447,5 \mathrm{~m} / \mathrm{min}$ y $\mathrm{VB} \approx 0,18 \mathrm{~mm}$.

Las irregularidades mostradas en el perfil de rugosidad obtenido durante el fresado con el portaherramientas PH1 y velocidad de corte de 447,5 m/min (Figura 6b) tienen su origen en el aumento del valor máximo de $\mathrm{F}_{\mathrm{Y}}$ como se observa en la Figura 8b, lo que origina un mayor desplazamiento en Y y una mayor deflexión radial.

Durante el fresado con velocidades de corte de $383,2 \mathrm{~m} / \mathrm{min}$, los mejores resultados en cuanto a rugosidad superficial se obtuvieron con el portaherramientas de acero PH1, como se observa en las Figuras 5a, 8 y 9. El material de este portaherramientas tiene menor densidad, rigidez mecánica y modulo Young del material del portaherramientas PH2 que está fabricado de metal duro. Sin embargo, de acuerdo a lo analizado anteriormente, el fresado con este portaherramientas es más estable debido a que el armónico 3 de la frecuencia de entrada de dientes está más alejado de la frecuencia modal 1

El perfil de rugosidad superficial mostrado en la Figura 8a, fue obtenido para la condición más estable durante el mecanizado de la pieza con $\mathrm{V}_{\mathrm{C}}=383,2 \mathrm{~m} / \mathrm{min}(\mathrm{f} 3=821,4 \mathrm{~Hz})$ y empleando el portaherramientas de metal duro PH1. Se evidencia que para estas condiciones se obtiene un proceso de corte estable con marcas regulares en la superficie de la pieza con una separación correspondiente al valor del avance por revolución del husillo. Estos perfiles de rugosidad presentan una uniformidad en el espaciamiento de los picos de rugosidad y en su altura, semejantes a los observados en la figura $6 \mathrm{a}\left(\mathrm{V}_{\mathrm{C}}=447,5 \mathrm{~m} / \mathrm{min} ; \mathrm{n}=9583\right.$ rpm; $\mathrm{f} 3=958,3 \mathrm{~Hz}$ ), lo que demuestra que esto es propio de condicione estables.

En el perfil de rugosidad mostrado en la Figura $8 \mathrm{~b}\left(\mathrm{~V}_{\mathrm{C}}=383,2 \mathrm{~m} / \mathrm{min} ; \mathrm{f} 3=821,4 \mathrm{~Hz}\right.$; portaherramientas PH2) se evidenció que las marcas dejadas por la herramienta de corte no se corresponden con los valores del avance, siendo irregulares en toda la longitud de medición de la rugosidad con valores aproximados a 4 veces el valor del avance por dientes, por lo que en la superficie de la pieza quedan marcas irregulares produciéndose mayores asperezas en la topografía de la misma. Estos resultados indican que el proceso de fresado para esta condición es inestable como se observa en la figura.

Nexo Revista Científica / Vol. 29, No. 02, pp. 69-82 / Diciembre 2016 


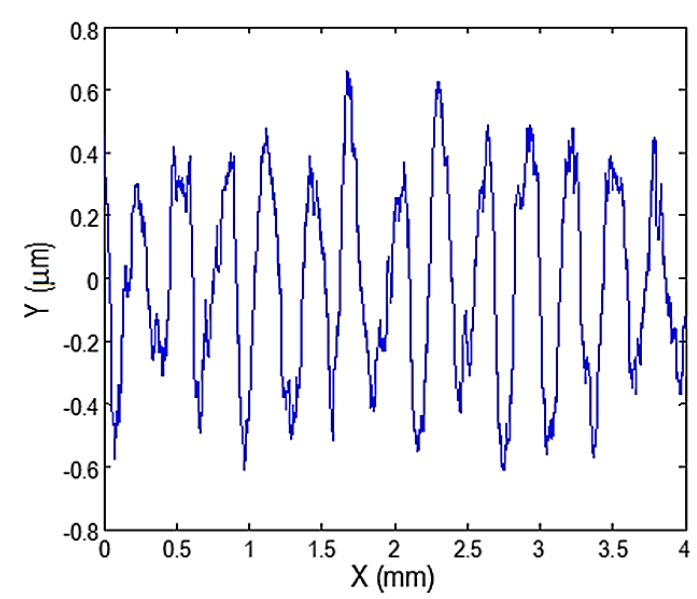

a)

$\mathrm{Ra}=0,27 \mu \mathrm{m}$

$$
\mathrm{Rq}=0,34 \mu \mathrm{m}
$$

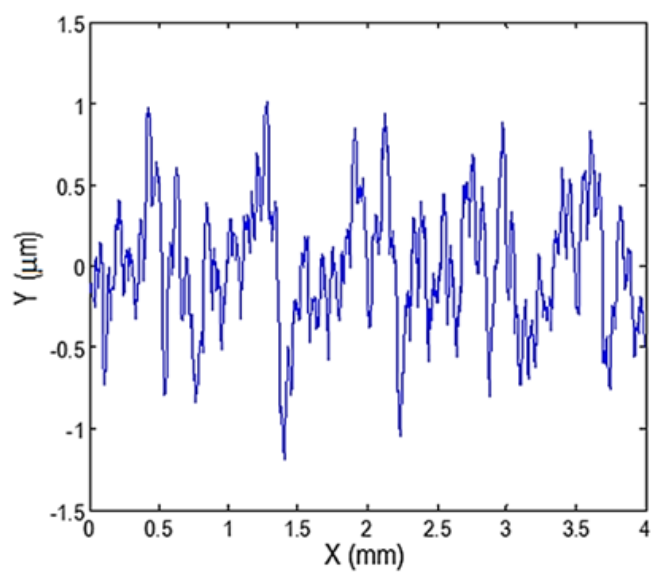

b)

$$
\mathrm{Ra}=0,27 \mu \mathrm{m} \quad \mathrm{Rq}=0,34 \mu \mathrm{m}
$$

Figura 8. Perfil de rugosidad en el sentido del avance. a) Portaherramientas $\mathrm{PH} 2$. $\mathrm{V}_{\mathrm{C}}=383,2 \mathrm{~m} / \mathrm{mim}$. b)

Portaherramientas PH1. $\mathrm{V}_{\mathrm{C}}=383,2 \mathrm{~m} / \mathrm{mim}$.

Al comparar las señales de fuerza en el dominio del tiempo obtenidas durante el fresado con velocidad de corte de 383,2 m/min (Figuras 9a y 9b) se observa que la señal de $F_{Y}$ obtenida para el portaherramientas PH1 tiene menor amplitud que la obtenida para el portaherramientas PH2, verificándose además que la deflexión radial durante el fresado con el portaherramientas PH1 también es menor por lo que los desplazamientos en el eje Y también son menores, generándose de esta forma perfiles de rugosidad más uniformes debido a una mayor estabilidad dinámica del sistema.

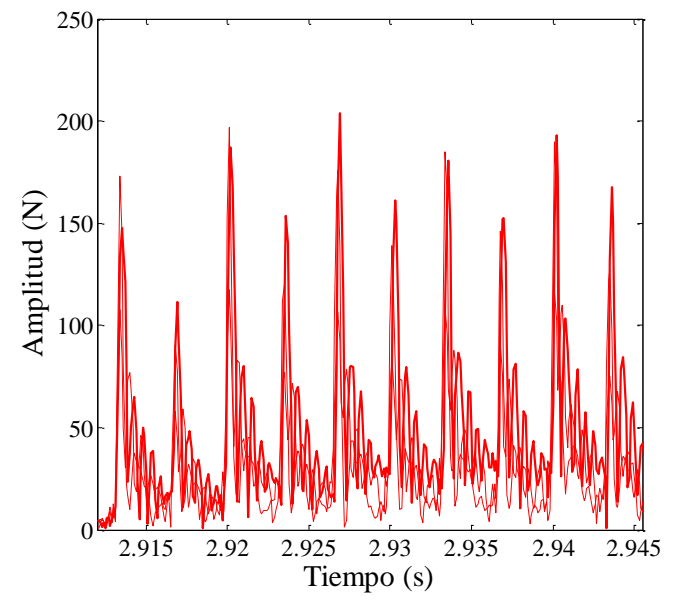

a)

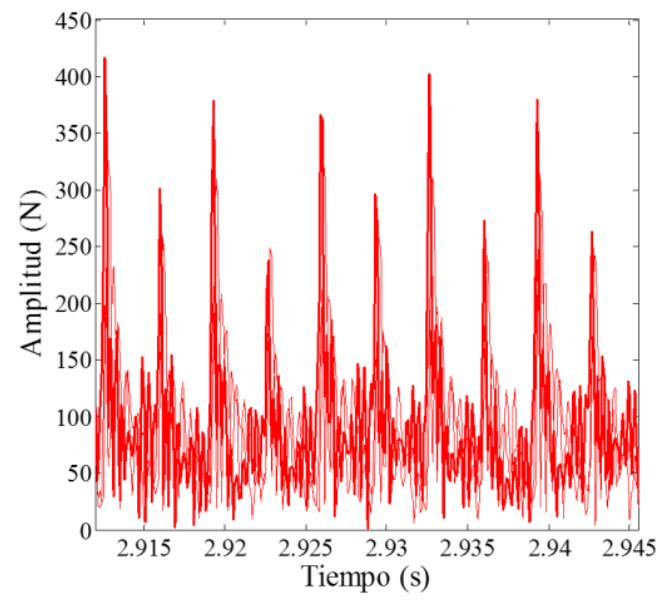

b)

Figura 9. Señales de fuerza en el dominio del tiempo. a) Portaherramientas $\mathrm{PH} 1 . \mathrm{V}_{\mathrm{C}}=383,2 \mathrm{~m} / \mathrm{min}$ y $\mathrm{VB} \approx 0,18$ mm. b) Portaherramientas $\mathrm{PH} 2 . \mathrm{V}_{\mathrm{C}}=383,2 \mathrm{~m} / \mathrm{min}$ y $\mathrm{VB} \approx 0,18 \mathrm{~mm}$.

En la Figura 11a se muestran los valores de la Raíz Media Cuadrática $(R M S)$ de las señales de fuerza en el dominio del tiempo obtenidas durante el fresado don velocidad de corte de 447,5 m/min. En la misma se observa que las $R M S$ de las componentes Fx, Fy y Fz de la fuerza de corte cuando se mecaniza con el portaherramientas $\mathrm{PH} 2$, tienen menores valores, lo que indica que las señales obtenidas tienen menor energía que las señales obtenidas durante el mecanizado con el portaherramientas PH1.

Nexo Revista Científica / Vol. 29, No. 02, pp. 69-82 / Diciembre 2016 


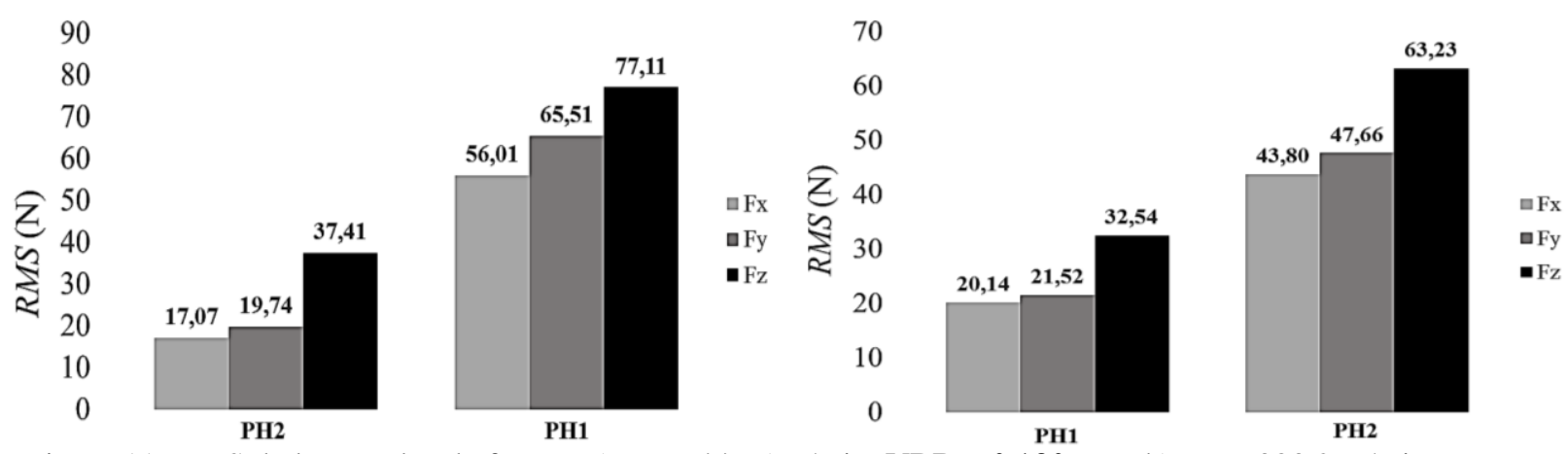

Figura 11. RMS de las señales de fuerza. a) $\mathrm{V}_{\mathrm{C}}=447,5 \mathrm{~m} / \mathrm{min}$. $\mathrm{VBB} \approx 0,180 \mathrm{~mm}$. b) $\mathrm{V}_{\mathrm{C}}=383,2 \mathrm{~m} / \mathrm{min}$. $\mathrm{VBB} \approx$ $0,180 \mathrm{~mm}$.

Las $R M S$ de las fuerzas obtenidas durante el fresado con velocidad de corte de $383,2 \mathrm{~m} / \mathrm{n}$, Figura $11 \mathrm{~b}$ indican que las componentes de la fuerza de corte, para esta condición de corte, tuvieron menor energía cuando se mecanizó con el portaherramientas de acero (PH1), corroborando de esta forma que, para esta condición el proceso fue dinámicamente más estable.

\section{CONCLUSIONES}

Partiendo del análisis de los resultados experimentales se concluyó que la rugosidad superficial en el fresado de superficies inclinadas con altas velocidades de corte depende en mayor medida de la estabilidad dinámica del proceso de corte que de las velocidades de corte y de avance.

Se comprobó que en el fresado de superficies inclinadas, cuando el proceso es estable, se obtienen menores valores de rugosidad superficial y perfiles de rugosidad uniformes en espaciamiento y altura, por lo que se corrobora que estas dos variables se pueden utilizar para evaluar la estabilidad dinámica en un proceso de fresado de superficies inclinadas con altas velocidades.

Se verificó que el aumento de los valores de la componente $\mathrm{F}_{\mathrm{Y}}$ de la fuerza de corte y la $R M S$ de las componentes $F_{X}, F_{Y}$ y $F_{Z}$ de la señal obtenidos durante el proceso de fresado de superficies inclinadas de acero endurecido AISI D6 indican un incremento de la instabilidad dinámica, produciendo un aumento de los valores de la rugosidad superficial y de las irregularidades del perfil de rugosidad.

\section{REFERENCIAS}

Antonialli, A., Diniz A. y Pederiba. R. (2010). Vibration analysis of cutting force in titanium alloy milling. International Journal of Machine Tools and Manufacture, 50(1), 65 - 73.

Bediaga, I., Hernández, J., Muñoa, J. y Uribe-Etxeberria, R. (2004) Influencia de las técnicas de variación de velocidad de cabezal en la estabilidad del proceso de fresado: estudio comparativo. XV Congreso de Máquinas-Herramienta y Tecnologías de Fabricación, Oct 2004, Donostia - San Sebastian, Spain. pp. 687702.

Cao, Z., Li, H., Yin, Z. y Liu, W. (2014) Analysis and Prediction of Micromilling Stability with Variable Tool Geometry. Hindawi Publishing Corporation Advances in Mechanical Engineering, Volume 2014, 6 p.

Cosma, M. (2011). Experimental studies on influence of tool path in 3-axes B.N.E.M. on inclined surfaces at 45 degrees. Academic Journal of Manufacturing Engineering, 9(4), 30-35.

Nexo Revista Científica / Vol. 29, No. 02, pp. 69-82 / Diciembre 2016 


\section{Y. Sánchez-Hechavarría y M. Mariño Cala}

Diniz, A.; Marcondes, F. y Coppini, N. (2010). Tecnologia da usinagem dos metais. São Paulo. Brasil: $7^{\text {ma }}$ ed. Artileber Editora Ltda.

Efstathiou, C., Vakondios, D., Chatzikokolaki, A. y Antoniadis A. (2014). Optimal cutting conditions in ball-end milling of complex surfaces taking into account the desired surface roughness. International Journal of Advancements in Mechanical and Aeronautical Engineering, 1(3), 126-130.

Ganguli, A., Deraemaeker, A., Romanescu, I., Horodinca, M. y Preumont, A. (2006). Simulation and Active Control of Chatter in Milling via a Mechatronic Simulator. Journal of Vibration and Control, 12(8), 817848.

Gok, A., Gologlu, C., Demirci, I. H. y Kurt, Mustafa. (2012). Determination of Surface Qualities on Inclined Surface Machining with Acoustic Sound Pressure. Journal of Mechanical Engineering, 58(10), 587-597.

Grossi, N., Sallese, L., Scippa, L. y Campatelli, G. (2014), Chatter stability prediction in milling using speed-varying cutting force coefficients. 6th CIRP International Conference on High Performance Cutting, Procedia CIRP 14. 170-175.

Haythem, G. (2011), Analysis and modeling of depth-of-cut during end milling of deposited material. F. W. Frank Liou (Tutor). Tesis de maestría. Universidade Missouri University of Science and Technology. Estados Unidos. 68 p.

Ko, J. H. \& Shaw. K. C. (2009). Chatter prediction based on frequency domain solution in CNC pocket milling. International journal of precision engineering and manufacturing, 10(4), 19-25.

Lamraoui, M., Barakat, M., Thomas, M. y El Badaoui, M. (2015). Chatter detection in milling machines by neural network classification and feature selection. Journal of Vibration and Control, 21(7), 1251-1266.

Mandal, S. (2014). Applicability of Tool Condition Monitoring Methods Used for Conventional Milling in Micromilling: A Comparative Review. Hindawi Publishing Corporation. Journal of Industrial Engineering, Vol. 2014. 8 p.

Mendes de Aguiar, M., Diniz, A. y Pederiva. R. (2015). Correlating surface roughness, tool wear and tool vibration in the milling process of hardened steel using long slender tools. International Journal of Machine Tools \& Manufacture, 68, 1-10.

Pal-Pandian, P., Prabhu-Raja, V. y Sakthimurugan, K. (2012). Identification of stability lobes in high-speed machining of thin ribs. International Journal of Engineering and Science, 1(8), 01-06.

Pivetta, C. (2005). Uma contribuição aoestudo do fresamento de aço endurecido com fresa de topo esférico. Anselmo Eduardo Diniz (Tutor). Tesis de maestría. Universidade Estadual de Campinas. Brasil. 130 p.

Polli, M. (2005). Análise da estabilidade dinâmica do processo de fresamento a altas velocidades de corte. Walter Lindolfo Weingaertner (Tutor). Tesis de doctoral. Universidade Federal de Santa Catarina. Brasil. $214 \mathrm{p}$.

Quintana, G. (2009). Stability lobes diagram identification and surface roughness monitoring in milling processes. Joaquim de Ciurana i Gay (Tutor). Tesis doctoral. Universidad de Girona. España. 2009. 113 p.

Nexo Revista Científica / Vol. 29, No. 02, pp. 69-82 / Diciembre 2016 


\section{Y. Sánchez-Hechavarría y M. Mariño Cala}

Toh, C. (2004). Surface topography analysis in high speed finish milling inclined hardened steel, Precision Engineering, 28(4), 386-898.

Wojciechowski, S. (2011). Machined surface roughness including cutter displacements in milling of hardened steel. Metrology and measurement systems, 18(3), 429-440.

Wojciechowski, S., Twardowski, P. y Chwalczuk, T. (2013). Surface roughness of hardened steel after ball end milling with various surface inclination. Archives of mechanical technology and automation, 33(1), 5564.

Yue, J. (2006) Creating a Stability Lobe Diagram. Proceedings of the 2006 IJME- INTERTECH Conference, Session IT, 301-050.

\section{SEMBLANZA DE LOS AUTORES}

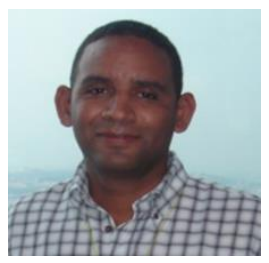

Yanier Sánchez-Hechavarría se graduó de Ingeniero Mecánico en la universidad de Oriente, Cuba en 2003. Cursó la Maestría de Procesos de Manufactura y Materiales de la Universidad de Oriente en el año 2007. Ha desarrollado estudios de doctorado en la Universidad Estadual de Campinas en Brasil y en la Universidad de Oriente sobre el tema que da contenido a este artículo. Su área de investigación es el mecanizado y micromecanizado con altas velocidades de corte. Actualmente es Profesor Auxiliar del Departamento de Manufactura y Materiales de la Universidad de Oriente.

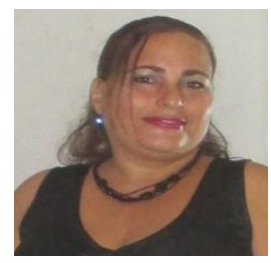

Maritza Mariño-Cala se graduó de Ingeniera Metalúrgica en el Instituto Superior Minero Metalúrgico de Moa, Holguín, en 1995. Defendió el grado de Master en Ciencias en Metalurgia Extractiva en el año 1999. Recibió entrenamientos de postgrado en la Universidad de Gante, Bélgica en el 2000 y en la Universidad de Patras y el Centro Helénico de Investigaciones Metalúrgicas desde el 2004 hasta el 2006. Defendió el grado de Doctor en Ciencias Técnicas en la Especialidad de Metalurgia en el 2009. Es profesora Titular del Departamento de Manufactura y Materiales de la Universidad de Oriente. Sus áreas de investigación son los materiales compuestos y los procesos de mecanizado con altas velocidades de corte.

Nexo Revista Científica / Vol. 29, No. 02, pp. 69-82 / Diciembre 2016 